\title{
Entwicklung von galvanisch gekoppelten Gleitlagern zur Reduzierung von Reibung und Verschleiß
}

\author{
T. Amann' ${ }^{10} \cdot$ W. Chen ${ }^{2}$ M. Baur ${ }^{1}$ A. Kailer ${ }^{1}$ J. Rühe² \\ Eingegangen: 29. März 2019 / Angenommen: 5. September 2020 / Online publiziert: 28. September 2020 \\ (c) Der/die Autor(en) 2020
}

\section{Zusammenfassung}

Durch Reibung und Verschleiß werden weltweit Gesamtkosten von $250 \mathrm{Mrd}$. $€ / \mathrm{Jahr}$ verursacht und $8120 \mathrm{MtCO}_{2}$-Emissionen freigesetzt. Die aktuellen Herausforderungen bestehen darin, die Vorteile ultraniedriger Reibung, der verschleißlosen Gleitund Reibungskontrolle sowie der wasserbasierten Schmierung hinsichtlich Energie- und Ressourceneffizienz für technische Anwendungen zu nutzen. An dieser Problematik, der Steigerung der Energieeffizienz und Nachhaltigkeit, setzt diese Arbeit an. Vorarbeiten haben gezeigt, dass mit speziellen mesogenen Flüssigkeiten Superlubrizität $(\mu<0,005)$ realisiert werden kann. In dieser Arbeit sollen neben der anwendungsnahen Prüfung dieser mesogenen Fluide auch die Eigenschaften von Wasser als Schmierstoff durch Additivierung mit komplexen Fluiden (ionischen Flüssigkeiten, lyotroper Flüssigkristall) und Einbringung eines elektrochemischen Schutzes durch galvanische Kopplung verbessert werden. Durch die Zugabe der komplexen Fluide in Wasser wurden Reibung- und Verschleiß in Modellreibversuchen verbessert. Gleitlagerversuche mit dem mesogenen Schmierstoff zeigen bei galvanisch induziertem Oberflächenpotenzial, durch Kopplung des Stahllagers mit Kupfer, eine Reibwertreduzierung um $60 \%$ und eine Verschleißreduktion um $40 \%$ im Vergleich zu einem Referenzöl.

\section{Development of galvanically coupled plain bearings to reduce friction and wear}

\begin{abstract}
Friction and wear cause total costs of 250 billion Euros/year worldwide and release $8120 \mathrm{MtCO}_{2}$ emissions. The current challenges are to exploit the advantages of ultra-low friction, wear-free sliding and friction control and water-based lubrication in terms of energy and resource efficiency for technical applications. This thesis deals with the issue of increasing energy efficiency and sustainability. Preliminary work has shown that super-lubricity $(\mu<0.005)$ can be achieved with special mesogenic fluids. Besides the application-oriented testing of these mesogenic fluids, this work also aims at improving the properties of water as a lubricant by adding complex fluids (ionic liquids, lyotropic liquid crystals) and introducing electrochemical protection by galvanic coupling. The addition of complex fluids to water improved friction and wear in model friction tests. Plain bearing tests with the mesogenic lubricant show a friction coefficient reduction of $60 \%$ and a wear reduction of $40 \%$ compared to reference oil at galvanically induced surface potential due to the coupling of the steel bearing with copper.
\end{abstract}

\section{Problemstellung und Lösungsansatz}

Die Reduktion von Reibung (Energieeffizienz) und Verschleiß (Nachhaltigkeit) in technischen Anlagen spielen bei

T. Amann

tobias.amann@iwm.fraunhofer.de

1 Fraunhofer-Institut für Werkstoffmechanik IWM, Wöhlerstr. 11, 79108 Freiburg, Deutschland

2 Albert-Ludwigs-Universität Freiburg, Freiburg, Deutschland der Realisierung der geforderten ökonomischen, ökologischen und gesellschaftspolitischen Ziele eine zentrale Rolle. Durch Reibung und Verschleiß werden weltweit Gesamtkosten von $250 \mathrm{Mrd}$. $€ / \mathrm{Jahr}$ verursacht und $8120 \mathrm{MtCO}_{2}-$ Emissionen freigesetzt [1]. Die aktuellen Herausforderungen bestehen darin, die enormen Vorteile ultraniedriger Reibung, der verschleißlosen Gleit- und Reibungskontrolle sowie der wasserbasierten Schmierung hinsichtlich Energie- und Ressourceneffizienz für technische Anwendungen zu nutzen [2]. An dieser Problematik, der Steigerung der Energieeffizienz und Nachhaltigkeit („Green-Tribology“), 
setzt diese Arbeit an. Stand der Technik ist es, spezielle, auf die jeweiligen Anforderungen abgestimmte Schmierstoffe auf Mineralölbasis einzusetzen. Die Produktion von Mineralöl hat während den letzten 20 Jahren zugenommen. In Deutschland werden etwa eine Million Tonnen Schmierstoffe verwendet, von denen nur etwa die Hälfte des Altöls verwertet und entsorgt wird [3]. Diese große Menge an eingesetzten Schmierstoffen ist mit hohen Kosten bei der Förderung, Herstellung und Entsorgung verbunden und kann zusätzlich eine Belastung für die Umwelt darstellen. Eine Möglichkeit diese Probleme zu lösen besteht in der Substitution dieser toxisch bedenklichen und aufwändig zu entsorgenden Schmierstoffe auf Mineralölbasis durch biologisch inspirierte Schmierstoffe auf Wasserbasis. In diesem Kontext werden unterschiedliche neuartige Additive wie ionische Flüssigkeiten (ILs: ionic liquids), mesogene Fluide (MF: mesogenic fluids) und ionische Flüssigkristalle (ILCs: ionic liquid crystals) diskutiert [4]. Eigene Vorarbeiten haben gezeigt, dass mit speziellen MFs tribologische Eigenschaften verbessert werden können, bis hin zu Realisierung ultraniedriger Reibung auf Stahl $(\mu<0,005)$ [5-7] und auf diamantähnlichen Kohlenstoffschichten (diamond like carbon, DLC) [8]. ILs sind vielversprechende Additive, die aufgrund des ionischen Aufbaus starke Wechselwirkung mit Oberflächen zeigen. Die Eigenschaften von Wasser als Schmierstoff konnten durch Additivierung mit ILs [9] gezielt verbessert werden. Durch das Anlegen externer elektrischer Spannungen konnte Reibung und Verschleiß dieser wasserbasierten Fluide zusätzlich verbessert werden [10]. Es konnte außerdem gezeigt werden, dass mit reinen ILs Reibwerte durch Variation der elektrischen Spannung gezielt eingestellt werden können [11]. Ein Schritt zur technischen Ausnutzung dieser Wechselwirkung der ILs mit elektrochemischen Feldern konnte durch die Ausnutzung galvanisch induzierter Potenziale realisiert werden [12]. Mit dieser Methode entfällt eine externe Spannungsquelle und neben dem elektrochemischen Korrosionsschutz wird zusätzlich die Wechselwirkung der Ionen mit der Oberfläche erhöht, wodurch sich eine tribologische Schutzschicht aufbaut. Als weitere Additivklasse für einen wasserbasierten Schmierstoff wurden Alkylglucopyranoside, lyotrope Flüssigkristalle auf Zuckerbasis, tribologisch auf der Makro- und Nanoskala untersucht und vielversprechende Ergebnisse erzielt $[13,14]$. In diesem Projekt wurden unter anderem wasserbasierte Schmierstoffe mit speziellen oberflächenaktiven Molekülen verwendet. Bei wasserbasierten Schmierstoffen muss die Viskosität und Korrosivität von Wasser durch den Zusatz spezieller oberflächenaktiver Substanzen für tribologische Anwendungen angepasst bzw. verbessert werden. Der Mechanismus der als potenzialkontrollierte Reibung (potential-controlled friction, PCF) bezeichnet wird, wurde erstmals durch Chang et al. [15] eingeführt. Der Lösungsansatz in dieser Arbeit besteht

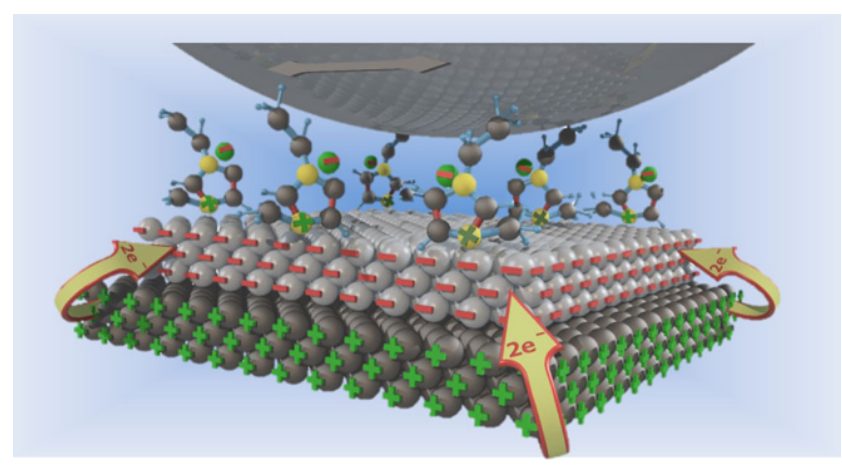

Abb. 1 Lösungsansatz: Galvanisch induzierte Potenziale auf der Oberfläche reduzieren korrosive Effekte und verstärken die Wechselwirkung mit den komplexen Fluiden (Bildung Tribolayer) die als Additiv dem Wasser beigesetzt sind

darin, galvanisch induzierte elektrochemische Potenziale auszunutzen, um zum einen tribochemische Reaktionen zu vermeiden (Korrosion) und zum anderen die Molekülorientierung an der Oberfläche zu verbessern (Abb. 1). Dadurch müssen keine externen Potenziale angelegt werden und das Prinzip wird für technische Anwendungen praktikabel. Durch die Kombination dieser Mechanismen soll ein möglichst dünner Schmierfilm (Dünnfilmschmierung, Thin-Film Lubrication, TFL) erzielt und stabilisiert werden. Dieses Reibregime, mit einer Schmierfilmdicke von einigen Nanometern bis zu einiger zehn Nanometern, liegt zwischen der elastohydrodynamischen Schmierung (EHL) und der Grenzschmierung. Wissenschaftliche Arbeiten haben gezeigt, dass dieses Reibregime der TFL zu minimalen Reibverlusten führt [16].

\section{Stand der Wissenschaft}

Die Forschungsarbeiten zu wasserbasierten Schmierstoffen mit oberflächenaktiven Substanzen und Graphen konzentrieren sich größtenteils auf den Bereich der Nanotribologie [17]. Es wurden mit solchen Substanzen extrem niedrige Reibwerte, sogenannte ,superlubricity“, nachgewiesen [18]. Diese Arbeiten liefern Grundlagen für das tiefere Verständnis der tribologischen Mechanismen, lassen sich aber nicht auf makroskopische, reale Reibkontakte übertragen. Tribochemische Reaktionen und galvanische Korrosionsvorgänge zwischen Edelstahl und einer DLC-Beschichtung bei Wasserschmierung wurden ebenfalls untersucht [19]. Einflüsse der molekularen Orientierung von ILs auf das tribologische Verhalten [20], sowie der Einfluss von $\mathrm{NaCl}$ in Wasser auf die molekulare Anordnung und die dadurch veränderten mechanischen Eigenschaften sind besonders hinsichtlich der Bildung eines Tribolayers interessant [21]. Bislang gibt es noch keine Forschungsarbeiten, die galvanisch induzierte elektrochemische Potenziale ausnutzen, um tribochemische Reaktionen zu beeinflussen. Bei For- 
schungsarbeiten, die sich mit der Untersuchung der makroskopischen Reibung beschäftigen, stehen Additive zur Optimierung von Wasser als Schmierstoff im Fokus. Dazu werden in wissenschaftlichen Arbeiten $\mathrm{MoS}_{2}$ Nanopartikel [22], CuO-beschichtete Nanopartikel [23], geschichtete Doppelhydroxid-Nanoplättchen [24], fluoriertes Graphen [25], Lithiumsalz und nichtionische Tenside [26], oxidierte aus Holz stammende Nanokohlenstoffe [27], Pflanzenextrakte [3] und ILCs [28] diskutiert. Es wurde herausgefunden, dass das Einlaufverhalten zum Erreichen extrem niedriger Reibung einen entscheidenden Einfluss bei der Verwendung einer wässrigen Mischung mit Schwefelsäure und Glycerin hat [29]. Nach dem Einlaufvorgang wird durch die Adsorption von Wasserstoffprotonen eine elektrische Doppelschicht gebildet, die eine abstoßende Doppelschichtkraft erzeugen kann, die einen Teil der Last trägt [29].

\section{Material und Methoden}

\subsection{Schmierstoffe}

Auf Grundlage eigener Vorarbeiten wurden vielversprechende Substanzen ausgewählt, die als Additiv in wasserbasierten Systemen eingesetzt werden können (Abb. 2). Es wurden neutrale Tenside auf Zuckerbasis (Alkylpolyglucoside - APG), formanisotropes 1,3-Diketon (mesogenes Fluid, MF-127070; Fa. Dr. Tillwich Werner Stehr) und ionische Flüssigkeiten (ILs, Fa. Iolitec) ausgewählt. Die Viskosität der Flüssigkeiten wurde mit einem Rotationsrheometer (Physica MCR 501, Anton Paar, Deutschland) mit Kegel-Platte-Geometrie (CP: 50-2/TG, Durchmesser: 49,915 mm, Winkel: 2,001 ${ }^{\circ}$ ) bestimmt. Das APG ist ein in der Natur vorkommender lyotroper Flüssigkristall, der aus einer hydrophoben Alkylkette mit 8 Kohlenstoffatomen (C8) und einer hydrophilen Kopfgruppe besteht. Diese Substanz kann in Lösung eine geordnete Struktur ausbilden und sich zusätzlich in einer Doppelschicht auf der Oberfläche anlagern. Es wurde eine Zugabe von gew. $40 \%$ von C8 zu deionisiertem Wasser gewählt (Viskosität: $29 \mathrm{mPa} \cdot \mathrm{s}$ bei $20^{\circ} \mathrm{C}$ ). MF-127070 wurde als Reinstoff verwendet (Viskosität: $12 \mathrm{mPa} \cdot \mathrm{s}$ bei $20^{\circ} \mathrm{C}$ ). Die ILs wurden aufgrund ihrer Mischbarkeit mit Wasser ausgewählt. Es wurde jeweils ei- ne 1 gew.\% IL zu deionisiertem Wasser hinzugegeben. Zusätzlich wurden als Benchmark 1 molare NaCl-Lösung und das Gleitlageröl WD40 (Viskosität: $2 \mathrm{mPa} \cdot \mathrm{s}$ bei $20^{\circ} \mathrm{C}$ ) verwendet.

\subsection{Tribologische Prüfungen}

Modellreibversuche wurden mit einem oszillierenden $\mathrm{Ku}-$ gel-Scheibe Tribometer (Fa. Optimol Instruments SRV-4, Abb. 3a) durchgeführt. Die Testparameter wurden auf $10 \mathrm{~N}$ Normalkraft, $1 \mathrm{~mm}$ Hub und $20 \mathrm{~Hz}$ Oszillationsfrequenz bei Raumtemperatur eingestellt. Jeder Test wurde dreimal mit einer Testdauer von 1,0h durchgeführt. Als Probenmaterial wurde Edelstahl SSt420 verwendet. Das elektrochemische Potenzial wurde durch eine gezielte Materialkombination induziert. Das dabei entstehende elektrische Potenzial wurde mit einem Potentiostat (Parstat4000, Fa. Ametek), bezogen auf eine Referenzelektrode $(\mathrm{Ag} / \mathrm{AgCl})$, gemessen (Abb. 3a). Zur Herstellung einer galvanischen Zelle wurden Kupfer $(\mathrm{Cu})$ als edleres und Aluminium $(\mathrm{Al})$ als unedleres Material im Gegensatz zu SSt420 verwendet. Reibversuche mit PTFE wurden als Referenz ohne galvanische Kopplung durch elektrische Isolation des Materials durchgeführt. Anwendungsnahe Reibversuche wurden mit dem Radialgleitlager-Tribometer (Abb. 3b) durchgeführt. Die rotierende Welle wird von einem Elektromotor (EC motor EC60 BL Y 400W KL 2WE A, Fa. maxon Motor) angetrieben. Die Welle ist beidseitig befestigt und rotiert in zwei Luftlagern (S304002 (40 mm I.D.), Fa. IBS Precision Engineering BV). Die Besonderheit dieses selbstgebauten Tribometers liegt in der In-situ-Erfassung des Verschleißes während des Reibversuchs. Diese Messung erfolgt direkt unterhalb des Lagers an der Halterung durch einen kapazitiven Sensor (HPB-40 Button Probe, Fa. Capacitec). Dadurch kann neben dem Reibwert auch das Einlauf- und Verschleißverhalten analysiert werden. Mit diesem Aufbau können Gleitlager ( $\varnothing_{\text {innen: }}$ 2-16 $\mathrm{mm}$ ) bei Normalkräften von 10-650 N (Zug-Druck-Kraftsensor Typ 8435, Fa. Burster) und bei Umdrehungen (Drehzahlen) von bis zu $3000 \mathrm{U} / \mathrm{min}$ getestet werden. Der Reibwert wird aus dem gemessenen Drehmoment (Drehmomentsensor TD110, Reaktionsmoment bis zu einem Nennmoment von $50 \mathrm{Nm}$ ) berechnet. Die Halterung für das zu prüfende Gleitlager wurde so konstruiert, dass eine galvanische Kopplung ermöglicht a

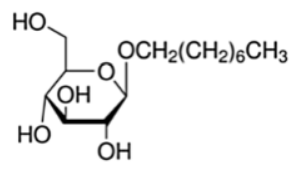

b

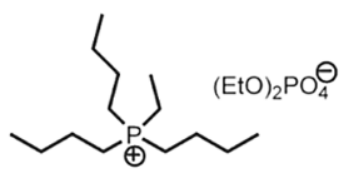

c

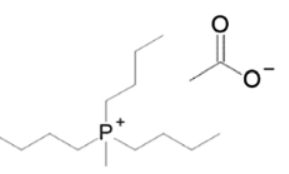

d

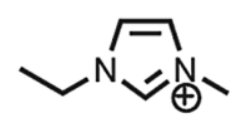

e

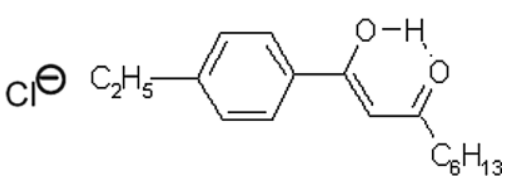

Abb. 2 Verwendete komplexe Fluide als Schmierstoff bzw. Additiv: a APG: Octyl $\beta$-D-Glucopyranosid (C8); b IL: Ethyltributylphosphonium diethyl phosphate (IN-0018-TG, [P2444] [DEP]); c IL: Tributylmethylphosphonium acetate (CS-0844, [P1444] [Oac]); d IL: 1-Ethyl-3-methylimidazoliumchlorid (IL-0093-HP, [EMIM][Cl]); e MF: 1-(4-ethyl phenyl)nonane-1,3-dione (MF-127070) 
a

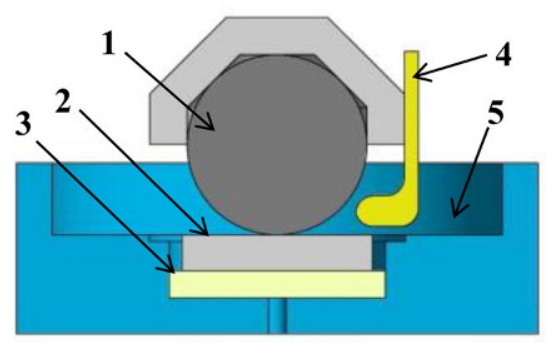

b

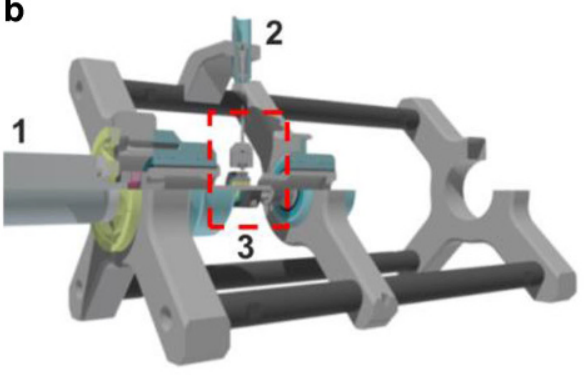

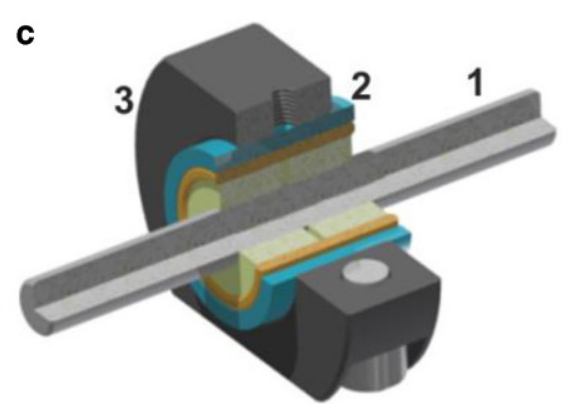

Abb. 3 a Modellreibversuch: Skizze des elektrisch isolierten Prüfaufbaus mit oszillierender Kugel-Scheibe Reibgeometrie: 1. Stahl-Kugel; Galvanische Zelle durch Materialkombination: 2. Obere Edelstahlscheibe; 3. Verwendete Materialien für die untere Scheibe: Kupfer (Cu), Aluminium (Al) und Teflon (PTFE); 4. Ag/AgCl-Referenzelektrode; 5. Elektrolyt- bzw. Schmierstoffreservoir. b Skizze des neu entwickelten Gleitlagerprüfstands mit In-situ-Verschleißmessung: 1. Motor zum Antrieb der Welle; 2. Schraube zum Einstellen der Normalkraft; 3. Galvanisch gekoppeltes Lager. Links und rechts vom Lager befinden sich Luftlager in denen die rotierende Welle gelagert ist. c Zu prüfendes Gleitlager: 1. Lagerwelle (grau); 2. Galvanische Kopplung mit Lagerring (grün), Kopplungselement (orange) und Isolation (blau); 3. Befestigung des Lagersystems. An der Unterseite dieser Halterung ist das Messsystem für die In-situ-Verschleißmessung angebracht

wird (Abb. 3c). Als Kopplungsmaterial wurden Aluminium (kathodisch), Kupfer (anodisch) und PTFE (isolierend) verwendet. Für die Reibversuche wurde eine Welle aus dem Stahl 100Cr6 mit einem Durchmesser von 7,98 mm verwendet. Die Sintereisenlager haben einen Innendurchmesser von $8,00 \mathrm{~mm}$ und eine Breite von $4,4 \mathrm{~mm}$. Für die Reibversuche wurden jeweils zwei Lager in der Halterung für die galvanische Kopplung verwendet. Bei der Versuchsdurchführung wurden Geschwindigkeitsrampen (StribeckKurven: $0-500 \mathrm{U} / \mathrm{min}, 0-0,21 \mathrm{~m} / \mathrm{s}, 2 \mathrm{~min}$ ) und Versuche bei konstanten Bedingungen (Dauerversuch: $500 \mathrm{U} / \mathrm{min}$, $0,21 \mathrm{~m} / \mathrm{s}, 30 \mathrm{~min}$ ) miteinander kombiniert. Ein Prüfzyklus umfasst dabei einen Dauerversuch und jeweils eine Stribeck-Kurve bei abnehmender und anschließend ansteigender Geschwindigkeit. Es wurden insgesamt 10 Zyklen (340 min, 150N, 2,1 MPa) durchlaufen.

\section{Ergebnisse und Diskussion}

\subsection{Modellreibversuche}

Es wurden mehrere komplexe Fluide (ILs, C8) und als Benchmark das Gleitlageröl (WD40) mit dem Edelstahl (SSt420) tribologisch untersucht. Durch Kopplung des

Tab. 1 Änderung Gesamtverschleiß durch Kopplung mit Aluminium und Kupfer im Vergleich zu PTFE

\begin{tabular}{lll}
\hline Schmierstoff & \multicolumn{2}{l}{ Verschleißänderung } \\
\cline { 2 - 3 } & $\begin{array}{l}\mathrm{Al} \\
\text { (in \%) }\end{array}$ & $\begin{array}{l}\mathrm{Cu} \\
\text { (in \%) }\end{array}$ \\
\hline $1 \%[\mathrm{P} 2444][\mathrm{DEP}]$ & -13 & 27 \\
$1 \%[\mathrm{P} 1444][\mathrm{Oac}]$ & -4 & 9 \\
$1 \mathrm{~mol} \mathrm{NaCl}$ & -2 & 26 \\
$1 \%[\mathrm{EMIM}][\mathrm{Cl}]$ & -75 & 16 \\
$40 \% \mathrm{C} 8$ & -66 & 41 \\
\hline
\end{tabular}

Stahls mit Aluminium wird bei allen untersuchten Zwischenstoffen ein kathodisches und bei Kopplung mit Kupfer ein anodisches elektrisches Potenzial induziert (Abb. 4a).

Bei Verwendung von $40 \%$ C8 wird als einziger Zwischenstoff eine niedrigere Reibarbeit als mit dem Vergleichsöl WD40 erzielt (Abb. 4b). Insgesamt wird die Reibarbeit durch die galvanische Kopplung nahezu nicht verändert. Im Gegensatz dazu ändert sich das Verschleißverhalten durch die galvanische Kopplung deutlich (Abb. 4c). Bei kathodischer Polarisierung werden mit $1 \%$ [EMIM] [Cl] und $40 \%$ C8 niedrigere Verschleißwerte als mit WD40 erzielt. Wie in Tab. 1 gezeigt, werden bei kathodischer Polarisierung mit Aluminium bei allen Schmierstoffen niedrigere - und bei anodischer Polarisierung mit Kupfer höhere - Verschleißwerte als ohne glavanische Kopplung mit PTFE erzielt. Der größte Unterschied bei der Verschleißänderung durch galvanische Kopplung im Vergleich zum unpolarisierten Zustand ist bei $40 \%$ C8 zu beobachten. Im Vergleich zu WD40 werden mit Ausnahme von $40 \%$ C8 deutlich höhere Verschleißwerte erzielt (Tab. 2). Die Ergebnisse zeigen, dass spezielle ionische Flüssigkeiten und besonders das Alkylpolyglucosid (40\% C8 in Wasser) sich als Additiv in Wasser in Kombination mit der galvanischen Kopplung eignen.

Tab. 2 Änderung Gesamtverschleiß bezogen auf WD40 gekoppelt mit Aluminium

\begin{tabular}{ll}
\hline Schmierstoff & $\begin{array}{l}\text { Verschleißänderung } \\
\text { (in \%) }\end{array}$ \\
\hline $1 \%[\mathrm{P} 2444][\mathrm{DEP}]$ & 111 \\
$1 \%[\mathrm{P} 1444][\mathrm{Oac}]$ & 396 \\
$1 \mathrm{~mol} \mathrm{NaCl}$ & 660 \\
$1 \%[\mathrm{EMIM}][\mathrm{Cl}]$ & 20 \\
$40 \% \mathrm{C} 8$ & -30 \\
\hline
\end{tabular}


Abb. 4 Ergebnisse der Modellreibversuche mit einer KugelPlatte-Geometrie $(10 \mathrm{~N}, 20 \mathrm{~Hz}$, $\mathrm{RT}, 1 \mathrm{~mm}, 1 \mathrm{~h}$ ) geschmiert mit Mischungen von deionisiertem Wasser mit Zugabe unterschiedlicher Additive (siehe horizontale Achse): a Induziertes Potenzial in Abhängigkeit des galvanischen Kopplungsmaterials und des verwendeten Zwischenstoffs; b Vergleich der Reibarbeit; c Vergleich des Verschleißvolumens von Kugel und Scheibe
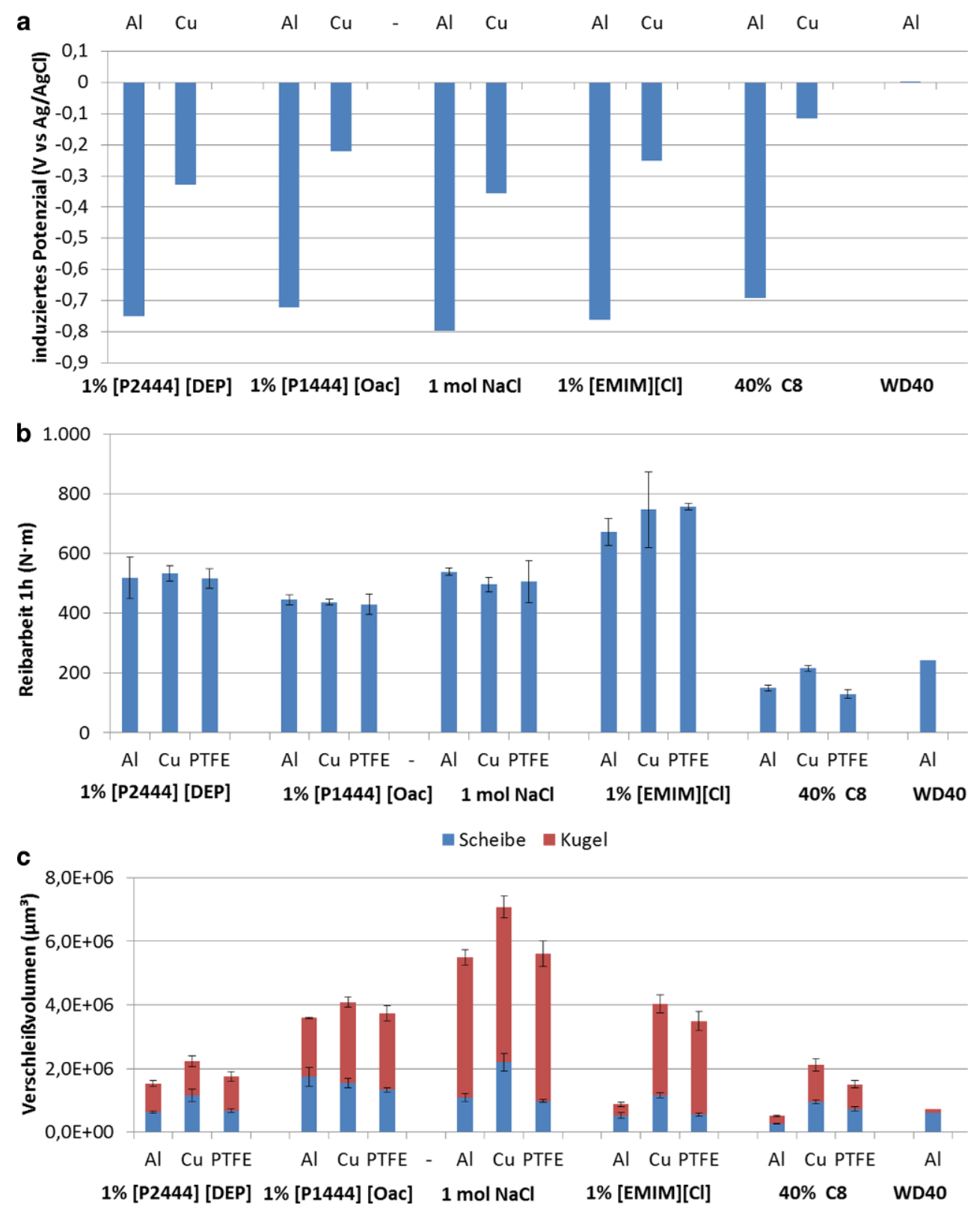

\subsection{Gleitlagerversuche mit galvanischer Kopplung}

Die Gleitlagerversuche wurden mit dem mesogenen Fluid MF-127070 [4] und der Mischung des lyotropen Flüssigkristalls von $40 \%$ C8 in Wasser [14] durchgeführt, da in den Vorarbeiten diese Substanzen als Schmierstoff die besten Ergebnisse zeigten. Bei der ersten Stribeck-Kurve bei ansteigender Geschwindigkeit zeigen, mit Ausnahme von 40\% C8 mit Kupfer, alle getesteten Schmierstoffe einen ähnlichen Reibwertverlauf (Abb. 5a). Der Reibwert sinkt von 0,2 bei langsamen Drehgeschwindigkeiten auf ca. 0,15 bei hohen Drehzahlen ab. Bei der letzten Stribeck-Kurve bei ansteigender Geschwindigkeit wird mit $40 \%$ C8 ein deutlich höherer Reibwert als bei der ersten Stribeck-Kur- ve erzielt (Abb. 5b). Mit dem Referenzöl und dem mesogenen Fluid werden ähnliche Reibwerte bei niedrigen Drehzahlen erreicht. Bei hohen Geschwindigkeiten zeigt MF127070 deutlich niedrigere Reibwerte $(\mu \approx 0,025)$ als das Referenzöl $(\mu \approx 0,1)$.

Während den Reibversuchen wurde der Reibwert (Abb. 6a) und der Verschleiß (Abb. 6b) in-situ aufgezeichnet. Die galvanische Kopplung wirkt sich bei den beiden oberflächenaktiven Substanzen, im Gegensatz zum Referenzöl, auf den Reibwert aus. Bei der Induktion eines kathodischen Potenzials werden bei MF-127070 und 40\% C8 niedrigere Reibwerte als bei Kopplung mit Kupfer erzielt. $40 \%$ C8 zeigt bei Kopplung mit Aluminium, mit Ausnahme des kurzzeitigen Anstiegs des Reibwerts, einen niedrige- 

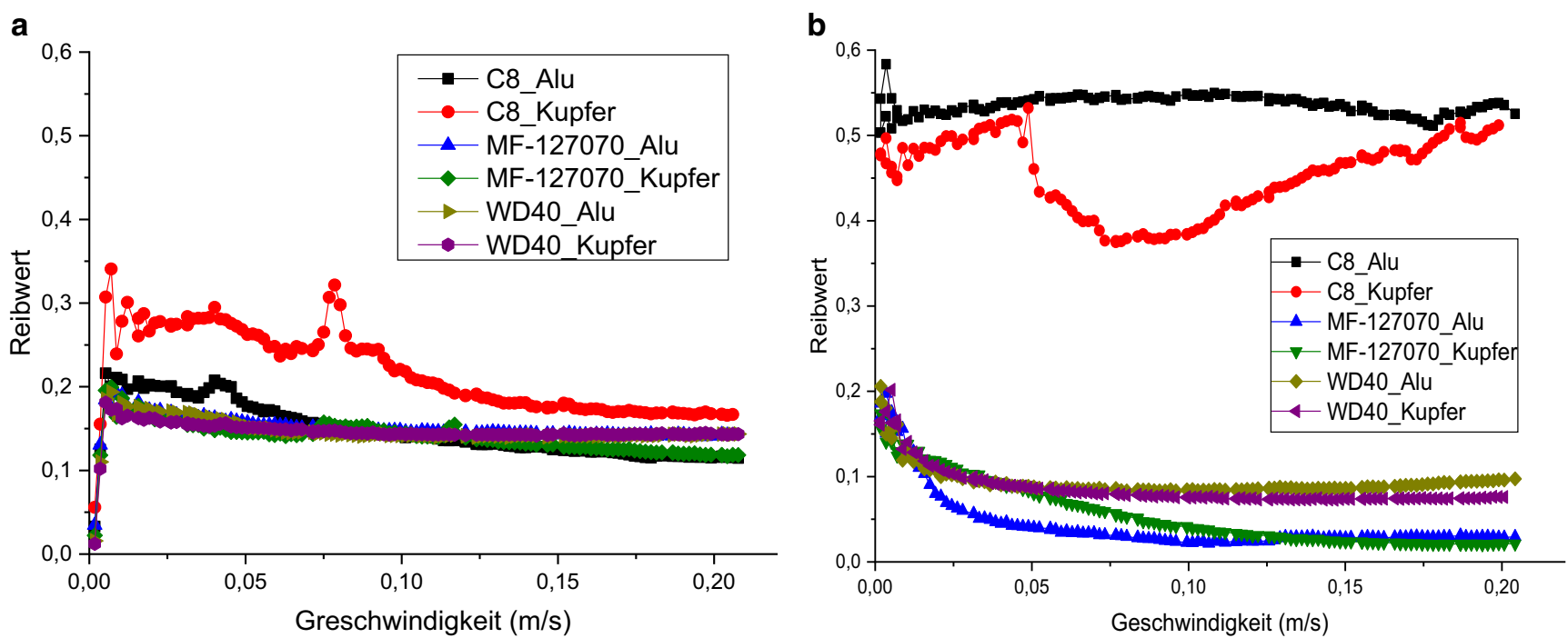

Abb. 5 Stribeck-Versuche: Reibwerte der Gleitlagerversuche mit Referenzöl WD40, mesogenem Fluid MF-127070 und lyotropen Flüssigkristall $40 \%$ C8 gekoppelt mit Aluminium und Kupfer: a Erste Stribeck-Kurve bei ansteigender Geschwindigkeit; b Letzte Stribeck-Kurve bei ansteigender Geschwindigkeit
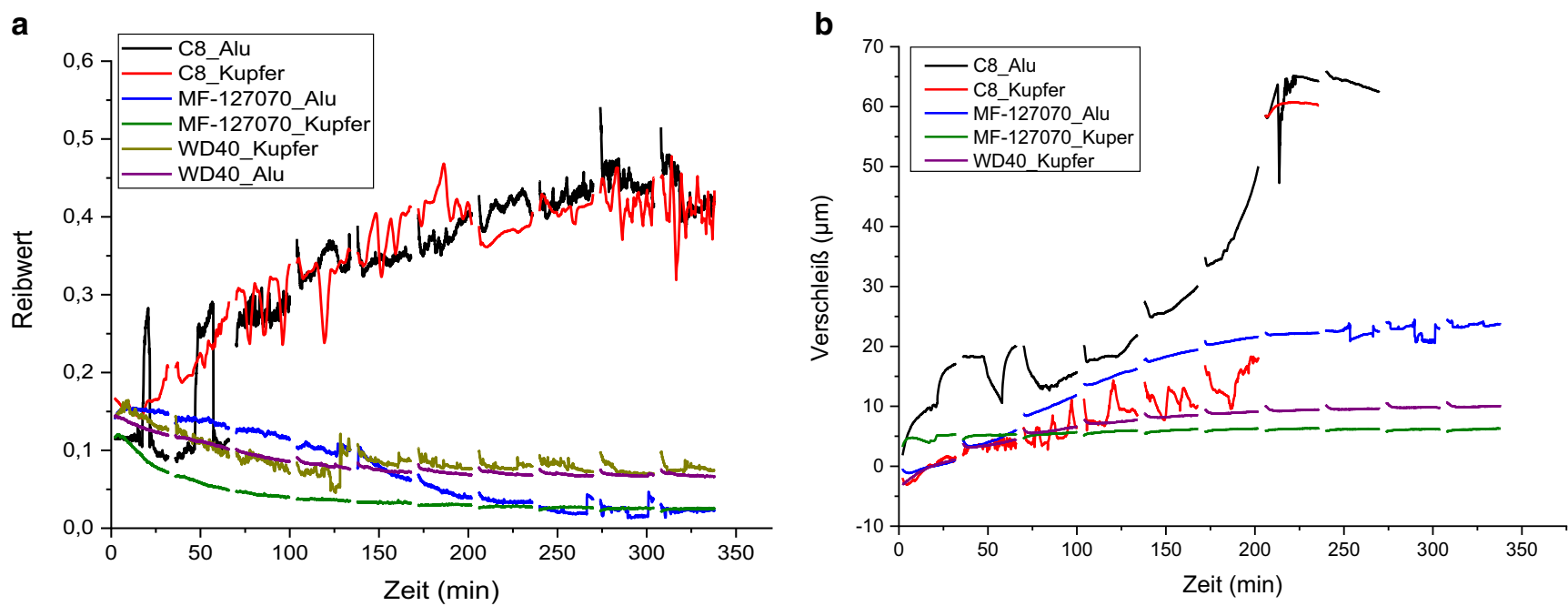

Abb. 6 Vergleich des Reibwerts (a) und der Verschleißentwicklung (b) während dem Dauerreibversuch mit Referenzöl WD40, mesogenem Fluid MF-127070 und lyotropen Flüssigkristall 40\% C8 galvanisch gekoppelt mit Aluminium und Kupfer (Die Ergebnisse mit MF-127070_Kupfer und WD40_Kupfer wurden während des Begutachtung online gestellt [30])

ren Reibwert als das Referenzöl. Dieser Reibwertanstieg ist vermutlich auf eine Phase der Mangelschmierung, und damit verbundenem Auftreten von stärkerer Mischreibung, zurückzuführen. Bis zum letzten Dauerversuch verändert sich das Reibverhalten der getesteten Fluide nochmal deutlich. Bei $40 \%$ C8 steigt der Reibwert auf ca. 0,45 an und verläuft bei Kopplung mit Kupfer sehr ungleichmäßig. Mit MF-127070 wird mit einem Reibwert von $\mu \approx 0,025$, unabhängig vom Kopplungselement, ein deutlich niedrigerer Reibwert als mit dem Referenzöl $(\mu \approx 0,07)$ erzielt. Bei den Versuchen mit dem Referenzöl WD40 bei Kopplung mit Aluminium war die In-situ-Verschleißmessung noch nicht installiert. Es wird aber vermutet, dass sich mit Aluminium als Kopplungsmaterial der Verschleiß nicht ändert, da sich aufgrund der elektrischen Isolation des Öls kein Potenzial aufbauen kann. Der Anstieg des Reibwerts bei Verwendung von $40 \%$ C8 korreliert sehr gut mit dem Verschleißverhalten. Bei Kopplung mit Aluminium steigt der Verschleiß kontinuierlich an, wobei mit Kupfer nach 200 min ein Anstieg des Verschleißes erfolgt. Beim Referenzöl WD40 wird nach ca. 150 min ein konstanter Verschleißwert erzielt. Dies korreliert ebenfalls gut mit dem konstanten Reibwert ab dieser Phase. Beim mesogenen Fluid MF-127070 wird bei Kopplung mit Aluminium ein konstanter Verschleißwert nach ca. 250 min erreicht. Im Gegensatz dazu bewirkt die anodische Polarisierung eine deutliche Verschleißredukti- 
Tab. 3 Übersicht der erzielten Reib- und Verschleißwerte

\begin{tabular}{llll}
\hline Schmierstoff & Galvanische Kopplung & Verschleiß $(\mu \mathrm{m})$ & Reibwert $^{\mathrm{a}}$ \\
\hline WD40 & Aluminium & - & 0,075 \\
WD40 & Kupfer & 10 & 0,066 \\
MF-127070 & Aluminium & 24 & 0,023 \\
MF-127070 & Kupfer & 6 & 0,026 \\
$40 \%$ C8 & Aluminium & 65 & 0,42 \\
$40 \%$ C8 & Kupfer & 60 & 0,42 \\
\hline
\end{tabular}

${ }^{a}$ Reibwert am Ende des letzten Dauerversuchs

on. In Tab. 3 sind die erzielten Reib- und Verschleißwerte zusammengestellt. Die galvanische Kopplung wirkt sich vor allem beim Verschleißverhalten von MF-127070 aus. Die Reibwerte werden durch die Kopplung nicht signifikant beeinflusst. Bei Reibwerten deutlich über 0,1 liegt Mischreibung vor und der Verschleiß, der sich dadurch einstellt, ist höher.

\section{Zusammenfassung und Ausblick}

In den Modellreibversuchen mit oszillierender KugelPlatte-Geometrie konnte der Einfluss der unterschiedlichen Oberflächenpolarisierung auf das Verschleißverhalten nachgewiesen werden. Durch Kopplung mit Aluminium (kathodisch) wurde der Verschleiß reduziert und mit Kupfer (anodisch) im Vergleich zum nicht polarisierten Zustand erhöht. Auf den Reibwert wirkt sich die Polarisierung nicht signifikant aus. Im Vergleich zum Referenzöl WD40 wurden nur mit dem lyotropen Flüssigkristall C8 $40 \%$ bei kathodischer Polarisierung niedrigere Verschleißwerte erzielt. Diese Methode der galvanischen Kopplung wurde anschließend erfolgreich auf ein selbstentwickeltes Gleitlagertribometer adaptiert. Bei diesem Tribometer können mehrere Gleitlager gleichzeitig unter identischen Prüfbedingungen getestet werden können. Durch die Implementierung einer In-situ-Verschleißanalyse ist es möglich das Einlauf- und Langzeitverhalten der Lager zu analysieren. Die Demonstration der Leistungsfähigkeit galvanisch gekoppelter Gleitlager erfolgte durch den Vergleich eines Referenzöls WD40 mit dem mesogenen Schmierstoff MF127070 (1-(4-ethyl phenyl)nonane-1,3-dione, Fa. Dr. Tillwich GmbH Werner Stehr) und einer Mischung aus $40 \%$ des lyotropen Flüssigkristall C8 (Tensid auf Zuckerbasis, Octyl $\beta$-D-Glucopyranosid) in Wasser. Die galvanische Kopplung erfolgte dabei wie bei den Modellversuchen mit Aluminium (kathodische Polarisierung) und Kupfer (anodische Polarisierung). Die Ergebnisse zeigen, dass mit MF-127070 bei Kopplung mit Kupfer extrem niedrige Reibungs- und Verschleißwerte erzielt werden können. Der Reibwert im Dauerversuch wird im Vergleich mit dem Referenzöl um $60 \%$ und der Verschleiß um $40 \%$ reduziert. Bei dem wasserbasierten Zwischenstoff $40 \%$ C8 deutet sich das Potenzial für tribologische Anwendungen bei der jeweils ersten Stribeck-Kurve und Dauerversuch an. Durch die Langzeitbelastung verdunstet Wasser, wodurch der Reibwert und der Verschleiß negativ beeinflusst werden. Diese Viskositätsänderung wurde nicht direkt gemessen, allerdings weist C8 eine deutlich höhere Viskosität als Wasser auf, wodurch Wasserverdunstung zu einem Anstieg der Viskosität führt [14]. Dieses Verhalten lässt sich dadurch erklären, da lediglich eine Mischung des Tensids C8 mit Wasser verwendet wurde und nicht wie bei MF-127070 ein vollständig additivierter und auf das Gleitsystem optimierten Schmierstoff. Zukünftige Arbeiten werden sich auf die Entwicklung eines wasserbasierten Schmierstoffs durch zusätzliche Additivierung des lyotropen Flüssigkristalls C8 konzentrieren.

Danksagung Wir danken dem Ministerium für Wirtschaft, Arbeit und Wohnungsbau Baden-Württemberg für die Finanzierung der Projekte BioSis und GLEX. Darüber hinaus danken die Autoren Susanne BeyerFaiß (Co. Dr. Tillwich GmbH Werner Stehr) und Dr. Maria Ahrens (Fa. Iolitec $\mathrm{GmbH}$ ) für die Kooperation in den Projekten.

Funding Open Access funding enabled and organized by Projekt DEAL.

Open Access Dieser Artikel wird unter der Creative Commons Namensnennung 4.0 International Lizenz veröffentlicht, welche die Nutzung, Vervielfältigung, Bearbeitung, Verbreitung und Wiedergabe in jeglichem Medium und Format erlaubt, sofern Sie den/die ursprünglichen Autor(en) und die Quelle ordnungsgemäß nennen, einen Link zur Creative Commons Lizenz beifügen und angeben, ob Änderungen vorgenommen wurden.

Die in diesem Artikel enthaltenen Bilder und sonstiges Drittmaterial unterliegen ebenfalls der genannten Creative Commons Lizenz, sofern sich aus der Abbildungslegende nichts anderes ergibt. Sofern das betreffende Material nicht unter der genannten Creative Commons Lizenz steht und die betreffende Handlung nicht nach gesetzlichen Vorschriften erlaubt ist, ist für die oben aufgeführten Weiterverwendungen des Materials die Einwilligung des jeweiligen Rechteinhabers einzuholen.

Weitere Details zur Lizenz entnehmen Sie bitte der Lizenzinformation auf http://creativecommons.org/licenses/by/4.0/deed.de.

\section{Literatur}

1. Holmberg K, Erdemir A (2019) The impact of tribology on energy use and $\mathrm{CO} 2$ emission globally and in combustion engine and electric cars. Tribol Int 135:389-396 
2. Urbakh M, Meyer E (2010) Nanotribology: the renaissance of friction. Nat Mater 9(1):8-10

3. Sagraloff N, Dobler A, Tobie T, Stahl K, Ostrowski J (2019) Development of an oil free water-based lubricant for gear applications. Lubricants 7:33

4. Amann T, Dold C, Kailer A (2013) Complex fluids in tribology to reduce friction: mesogenic fluids, ionic liquids and ionic liquid crystals. Tribol Int 65:3-12

5. Amann T, Kailer A, Beyer-Faiß S, Stehr W, Metzger B (2016) Development of sintered bearings with minimal friction losses and maximum life time using infiltrated liquid crystalline lubricants. Tribol Int 98:282-291

6. Amann T, Kailer A (2010) Ultralow friction of mesogenic fluid mixtures in tribological reciprocating systems. Tribol Lett 37: 343-352

7. Li K, Zhang S, Liu D, Amann T, Zhang C, Yuan C, Luo J (2018) Superlubricity of 1,3-diketone based on autonomous viscosity control at various velocities. Tribol Int 126:127-132

8. Amann T, Kailer A, Oberle N, Li K, Walter M, List M, Rühe J (2017) Macroscopic superlow friction of steel and diamond-like carbon lubricated with a formanisotropic 1,3-Diketone. ACS Omega 2(11):8330-8342

9. Kurz J, Amann T, Kailer A (2019) Tribological investigations of silicon nitride lubricated by ionic liquid aqueous solutions. Tribol Trans. https://doi.org/10.1080/10402004.2018.1549300

10. Amann T, Dold C, Kailer A (2016) Potential controlled tribological behavior of water-based ionic liquids. Key Eng Mater 674:250-256

11. Gatti F, Amann T, Kailer A, Abicht J, Rabenecker P, Baltes N, Rühe J (2019) Makroskopische Reibwertsteuerung durch elektrochemische Potentiale mit ionischen Flüssigkeiten. Tribol Schmierungstech 66(4-5):51-57

12. Amann T, Gatti F, Oberle N, Kailer A, Rühe J (2018) Galvanically induced potentials to enable minimal tribochemical wear of stainless steel lubricated with sodium chloride and ionic liquid aqueous solution. Friction 6(2):230-242

13. Chen W, Amann T, Kailer A, Rühe J (2020) Macroscopic friction studies of Alkylglucopyranosides as additives for water-based lubricants. Lubricants 8(1):11

14. Chen W, Amann T, Kailer A, Rühe J (2019) Thin-film lubrication in the water/octyl $\beta$-d-Glucopyranoside system: macroscopic and nanoscopic tribological behavior. Langmuir 35(22):7136-7145

15. Chang Q, Meng Y, Wen S (2002) Influence of interfacial potential on the tribological behavior of brass/silicon dioxide rubbing couple. Appl Surf Sci 202:120-125

16. Luo J, Wen S, Huang P (1996) Thin film lubrication. Part I. Study on the transition between EHL and thin film lubrication using a relative optical interference intensity technique. Wear 194:107-115
17. Duan Y, Liu Y, Zhang C, Chen Z, Wen S (2016) Insight into the tribological behavior of liposomes in artificial joints. Langmuir 32(42):10957-10966

18. Li J, Zhang C, Cheng P, Chen X, Wang W, Luo J (2016) AFM studies on liquid superlubricity between silica surfaces achieved with surfactant micelles. Langmuir 32(22):5593-5599

19. Alazizi A, Draskovics A, Ramirez G, Erdemir A, Kim SH (2016) Tribochemistry of carbon films in oxygen and humid environments: oxidative wear and galvanic corrosion. Langmuir 32(8):1996-2004

20. Watanabe S, Nakano M, Miyake K, Tsuboi R, Sasaki S (2014) Effect of molecular orientation angle of Imidazolium ring on frictional properties of Imidazolium-based ionic liquid. Langmuir 30(27):8078-8084

21. Khan SH, Kramkowski EL, Hoffmann PM (2016) Nacl-dependent ordering and dynamic mechanical response in Nanoconfined water. Langmuir 32(42): 10802-10807

22. Dong C, Yuan C, Wang L, Liu W, Bai X, Yan X (2016) Tribological properties of water-lubricated rubber materials after modification by MoS2 Nanoparticles. Sci Rep 6:35023

23. Yang P, Zhao X, Liu Y, Lai X (2016) Preparation and tribological properties of dual-coated $\mathrm{CuO}$ Nanoparticles as water based lubricant additives. j nanosci nanotechnol 16(9):9683-9689

24. Wang H, Liu Y, Chen Z, Wu B, Xu S, Luo J (2016) Layered double hydroxide Nanoplatelets with excellent tribological properties under high contact pressure as water-based lubricant additives. Sci Rep 6(22748):22748

25. Ye X, Ma L, Yang Z, Wang J, Wang H, Yang S (2016) Covalent Functionalization of Fluorinated Graphene and Subsequent Application as Water-based Lubricant Additive. ACS Appl Mater Interfaces 8(11):7483-7488

26. Wang Y, Yu Q, Cai M, Shi L, Zhou F, Liu W (2017) Synergy of lithium salt and non-ionic surfactant for significantly improved tribological properties of water-based fluids. Tribol Int 113:58-64

27. Kinoshita H, Nishina Y (2016) Investigations on tribological mechanisms of Graphene oxide and oxidized wood-derived Nanocarbons as water-based lubricating additives. Tribol Online 11(2):235-241

28. Avilés MD, Sánchez C, Pamies R, Sanes J, Bermúdez MD (2019) Ionic liquid crystals in tribology. Lubricants 7(9):72

29. Deng M, Li J, Zhang C, Ren J, Zhou N, Luo J (2016) Investigation of running-in process in water-based lubrication aimed at achieving super-low friction. Tribol Int 102:257-264

30. Amann T (2019) Blog, MikroTribologie Centrum $\mu$ TC. https:// www.mikrotribologiecentrum.de/de/mikrotribologiecentrum aktuelles/blogeintrag-01-03-2019.html. Zugegriffen: 3. Juni 2020 